\title{
Ultraviolet Imaging Observations of the cD Galaxy in Abell 1795:Further Evidence for Massive Starformation in a Cooling Flow
}

\author{
Eric P. Smith \\ Laboratory for Astronomy and Solar Physics, Code 681 - NASA/Goddard Space Flight \\ Center, Greenbelt, MD 20771 \\ Ralph C. Bohlin \\ Space Telescope Science Institute, Homewood Campus, Baltimore, MD 21218 \\ G. D. Bothun \\ Department of Physics \&5 Astronomy, University of Oregon, Eugene, OR 97403 \\ Robert W. O'Connell \\ Astronomy Department, University of Virginia, Charlottesville, VA 22903 \\ Morton S. Roberts \\ National Radio Astronomy Observatory, Edgemont Road, Charlottesville, VA 22903 \\ Susan G. Neff, Andrew M. Smith \& Theodore P. Stecher \\ Laboratory for Astronomy and Solar Physics, Code 680 - NASA/Goddard Space Flight \\ Center, Greenbelt, MD 20771
}




\begin{abstract}
We present images from the Ultraviolet Imaging Telescope of the Abell 1795 cluster of galaxies. We compare the cD galaxy morphology and photometry of these data with those from existing archival and published data. The addition of a far-UV color helps us to construct and test star formation model scenarios for the sources of UV emission. Models of star formation with rates in the range $\sim 5-20 M_{\odot} \mathrm{yr}^{-1}$ indicate that the best fitting models are those with continuous star formation or a recent $(\sim 4$ Myr old) burst superimposed on an old population. The presence of dust in the galaxy, dramatically revealed by HST images complicates the interpretation of UV data. However, we find that the broad-band UV/optical colors of this cD galaxy can be reasonably matched by models using a Galactic form for the extinction law with $E_{B-V}=0.14$. We also briefly discuss other objects in the large UIT field of view.
\end{abstract}

Subject headings: galaxies: individual, stellar content, cooling flows 


\section{Introduction}

The final fate of the cooling gas seen by $\mathrm{X}$-ray telescopes in clusters of galaxies has long been sought and any system which can provide evidence for star formation arising in a system of cooling gas is of great interest. The cD galaxy in Abell $1795(z=$ 0.0634 ) has been an object of much study since it was identified with the radio source 4C 26.42 (Merkelijn 1972) and later found to reside in a cluster cooling flow (McKee, et al. 1980). Subsequent optical studies (Sargent 1973, Heckman 1981) found it to possess extended nuclear emission-line gas $(d \sim 20 \mathrm{kpc}$, $\left.H_{o}=75, q_{o}=0.1\right)$. Spectroscopic studies in both the ultraviolet and optical reveal an unusual blue continuum ( $\mathrm{Hu}$ (1992), Allen 1995) whose structure has been recently traced in broad band optical imaging (McNamara \& O'Connell 1992). These properties have made it one of the best candidate galaxies for exhibiting star formation arising from a cluster cooling flow though other mechanisms can be invoked to explain the radio emission and emission-line gas.

Hubble Space Telescope observations, improved CCD sensitivity at blue wavelengths and the ultraviolet telescopes on board the Astro Space Shuttle payload have made this galaxy an object of renewed interest (McNamara \& O'Connell (1993), McNamara, et al. 1996a, Pinkney, et al. 1996). These recent obpervations have reveated regions with colors bluer than normal $((U-I) \sim 2.1$; McNamara \& O'Connell (1993) for an expected old stellar population, near the galaxy nucleus that may be the sites of recent star formation. These blue regions are confined to the very central $\left(r \lesssim 20^{\prime \prime}\right)$ region of the galaxy. The $\mathrm{cD}$ appearance at long wavelengths $(\lambda>7000 \AA)$ where the light is dominated by the oldest stars is smooth and does not suggest that a recent globally disturbing event (e.g. a major galaxy interaction) has occurred to trigger a burst of central star formation. Suggested possible triggers for the star formation have included radio jets, collision with a smaller galaxy (McNamara, et al. 1996a), and the interaction of the gas cooling out of the X-ray halo with the galaxy interstellar medium (ISM). Each of these interpretations predict some recent star formation in the galaxy nucleus, but with potentially differing morphologies, resultant population mixes and emission-line properties.

If the galaxy has undergone recent star formation then it is natural to turn to the ultraviolet (UV) part of the spectrum for observational confirmation. Young stars will most easily be distinguished from the preexisting stellar population by observations shortward of the $4000 \AA$ break. In particular, observations by the Ultraviolet Imaging Telescope (UIT) in its far-UV filter may be used as a valuable diagnostic for stars which are extremely hot $\left(T_{e f f} \gtrsim 10000 \mathrm{~K}\right)$. At the distance of $\mathrm{cD}(z=0.0634)$ as few as $1200 \mathrm{O}$ stars would be detectable with UIT (provided there was no obscuration by dust). At the wavelengths observed by the UIT all of the underlying old population (primarily $\mathrm{G}, \mathrm{K}$ and later type stars) will be invisible, with the important possible exception of extreme horizontal branch (EHB) stars (Ferguson, et al. 1991, Dorman, O'Connell, \& Rood 1995).

In section 2 we discuss the UIT data and photometry as well as archival data used in the analyses. We follow in section 3 with a discussion of the implications these new UV data have for star formation properties in Abell 1795. 


\section{Observational Data}

We present our image obtained with the UIT during the Astro-2 Space Shuttle mission of March 1995 in figure 1. The UIT took several exposures using a far-UV filter with $\lambda_{c}=1520 \AA ; \Delta \lambda=356 \AA$ (which we shall hereafter denote by the first two digits of its central wavelength). For this study we used only the deepest $(1310.5 \mathrm{sec})$ exposure. For a complete description of the instrument and preliminary data reduction steps involved see Stecher, et al. (1992). The UIT has a large field of view (40'diameter) so the entire cluster core was imaged, but little beyond the cD itself was detected. The nominal stellar point-spread function (PSF) for the UIT has a FWHM of 3 "', but the Abell 1795 inages have poorer resolution with FWHM of the stellar PSF of $\sim 6.8^{\prime \prime}$. Several factors can contribute to decreased resolution with the most common being crew motions onboard the shuttle during the observation. In section 2.3 we briefly discuss objects other than the $\mathrm{cD}$ in the UIT image.

We have also assembled a collection of archival data to complement our UIT observations of this cluster. These additional data, when combined with that from UIT create a unique, long wavelength lever arm with which to examine the star formation properties. We extracted two pipeline calibrated image data sets, taken with the F555W (HST "V") and F702W filters, from the Hubble Space Telescope (HST) archives (u2630401$6)$. After correcting the image headers for updated WFPC2 astrometry (J. Hu, private communication) we removed the cosmic rays and applied a charge transfer correction as outlined in Holtzman, et al. (1995) and combined the two longest exposure images (800 sec each). Since the portion of the $\mathrm{cD}$ galaxy seen by the UIT is the central $\sim 20^{\prime \prime}$ we used only that portion of the HST data (PC chip). No sky subtraction was performed on the HST images because the short exposure times $(800 \mathrm{sec})$ combined with small pixel size imply that there is little if any contribution to the F555W and F702W signal from a background.

B. McNamara kindly provided us with a copy of his calibrated $U$-band image obtained with the Kitt Peak National Observatory's $2.1 \mathrm{~m}$ telescope (McNamara \& O'Connell 1992). For comparing the global stellar population with that of the electrons responsible for the radio emission we acquired a copy of previously published VLA data from J.P. Ge (Ge \& ( wen 1993).

\subsection{Morphology}

One of the primary goals of the UIT was to investigate the changes in galaxy morphology with wavelength for a variety of galaxy types. As part of this effort we have constructed a series of figures comparing the UIT image of the $\mathrm{cD}$ galaxy with those from other passbands (figures 2 through 1 ). These figures show the UIT image resembles the $\mathrm{H} \alpha$ emission more nearly that the broad band optical emission and that the UV morphology has no obvious link to the radio morphology. The similarity of the UV and $\mathrm{H} \alpha$ morphologies prompted us to estimate the amount of $\mathrm{Ly} \alpha$ contamination in our UIT filter band. To determine the strength of any Ly $\alpha$ emission from the center of the galaxy we extracted an archival IUE spectrum of the $\mathrm{cD}$ galaxy ( $\mathrm{Hu}$, Cowie, \& Wang (1985)] and convolved it with the UIT filter response curve. Though the system does exhibit moderately strong Ly $\alpha$ emission (see $\$ 2.2$ ) this contributes little to the UIT measured flux. Moreover, Astro-2 Hop- 
kins Ultraviolet Telescope spectra show those emission-lines that are present in the UIT filter are weak and do not contribute substantially to the overall UV flux (Dixon, private communication). Thus, despite the superficial resemblance of the UIT $\mathrm{cD}$ image to the $\mathrm{H} \alpha$ morphology we conclude that the light is from a continuum source and not extended emission-line gas.

Direct comparison of the HST optical and UIT UV morphologies is complicated to interpret due to the great difference in resolution. However, the presence of the dust lane dramatically revealed in the HST data (Fig. 3) is reflected in the pinching of the UV contours in the UIT image. In general, the UV morphology is much less regular than the HST F555W and F702W morphologies. The blue lobes seen by McNamara \& O'Connell (1993) may be associated with the North and South peaks of the UV contours separated by $\sim 10^{\prime \prime}$, but we do not find these regions to be bluer than the galaxy as a whole (in $1520 \AA-V \equiv 15$ $V)$. The $3.6 \mathrm{~cm}$ and $1520 \AA$ morphologies of the $\mathrm{cD}$ galaxy are coaligned roughly NorthSouth (figure 1). McNamara \& O'Connell (1993) found the blue lobes at the ends of the radio emission leading them to posit that the jets may be inducing star formation via interaction with the galaxy ISM. The UV data suggest very weak concentrations in flux near the outer edges of the radio emission.

To further investigate the morphology of the UV light we compare it surface brightness profile (Fig. 5) with those at longer wavelengths We generated surface brightness profiles in the UV, F555W and F702W bands using the STSDAS ellipse task (Fig. 6), and fit a function to the profile in McNamara \& O'Connell (1993) for the $U$ profile. There is no evidence for a point source component to the UV surface brightness profile. We fit both elliptical ( $r^{\frac{1}{4}}$-law) and disk galaxy (exponential) light profiles to the UIT data and find that the disk galaxy light profile is a better match. Table 3.3 gives the results for our model galaxy profile fits. We also performed fits to the F702W profile. The best fit was achieved with a combined point source, exponential disk and $r^{\frac{1}{4}}$-law bulge model. However, the disk central surface brightness for this fit is more than 2 magnitudes brighter than the corresponding characteristic bulge surface brightness $\left(I_{e}\right)$ indicating that the central region $\left(r<8^{\prime \prime}\right)$ of the $\mathrm{cD}$ has a predominantly disk-like light distribution. Thus both the UV and visual light from the central portion of the galaxy are better characterized by exponential light distributions than by elliptical galaxy light distributions, but with the UV light being more extended $\left(r \sim 20^{\prime \prime}\right)$. Interestingly, Hu, Cowie, \& Wang (1985) found spectroscopic evidence for for emission-line gas rotation in the central part of the $\mathrm{cD}$ while Anton 1993 found no evidence for a rotating disk, but neither measurements were sensitive to the small spatial scales accessible to HST nor to the young stellar population easily isolated by the UIT.

The $V-R$ color index does not vary significantly over the central portion of the $\mathrm{cD}$. However, the (15- $V$ ) index does change with the central part being slightly redder than the outer parts. This is most likely do to the presence of the dust so easily visible in the HST image. O'Connell et al. (1992) found that several ellipticals observed with UIT generally become bluer with decreasing radius, but none of those galaxies were know to possess significant amounts of centrally concentrated dust. 


\section{2. cD Galaxy Photometry}

We performed our photometry using software specifically tailored to UIT data, but modeled on the DAOPHOT aperture photometry routine. Curve of growth analysis shows that the $\mathrm{cD}$ galaxy light (centered at $\alpha=13: 48: 52.42, \delta=+26: 35: 34.7)$ is contained within a circle of radius $\sim 25^{\prime \prime}$. The aperture magnitude and corresponding flux for the galaxy are $m_{15}=15.54 \pm 0.09(2.21 \times$ $\left.10^{-15} \mathrm{erg} \mathrm{s}^{-1} \mathrm{~cm}^{-2} \AA^{-1}\right)$. Henceforth, all pho-

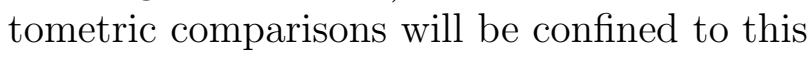
region or specific apertures within this radius. Using archival IUE spectra we are able to estimate the contribution to the UIT flux arising from the Ly $\alpha$ emission in the galaxy (see also $\mathrm{Hu}(1992))$. We have corrected the UIT magnitudes by $\Delta m_{15}=0.08$ to account for this emission-line contribution, yielding $m$ (continuum $)_{15} \equiv m_{c, 15}=15.62 \pm 0.09$. There was no detectable UV flux associated with the blue filamentary region described by McNamara \& O'Connell (1993). To check for the presence of the blue lobes we placed small $\left(5 \times 5^{\prime \prime}\right)$ apertures on the locations of lobes (from McNamara \& O'Connell (1993) figure 6 ) and on the galaxy center. We find no significant difference between the colors in these various regions. However, the low spatial resolution of the UIT image and typical uncertainty in its photometry $(\sim 0.1 \mathrm{mag})$ folded in with stochastic variations of the spatial distribution of O-stars imply that detecting color difference with these images would be difficult.

We photometered the HST and U band data within the same region and list the resulting magnitudes and colors in table 3.3. Steps outlined in Holtzman, et al. (1995) were taken to calibrate the HST photometry. For both the U and HST images we digitally re- moved a small elliptical galaxy at $r \sim 9.5^{\prime \prime}$ replacing the pixels with those from a region at the same radius, but 180 degrees different in position angle. The HST magnitudes were corrected for emission-line contributions using the data from Anton 1993. These corrections were small with $\Delta V=0.02$ and $\Delta R=0.06$ respectively. The only emission line in the $U$-band filter is the [O II] $\lambda 3727$ for which we correct by $\Delta U=0.04$. The optical magnitudes have been $K$-corrected using the models from Coleman, $\mathrm{Wu}, \&$ Weedman 1980. We estimated the $K$-correction for the $1520 \AA\left(K_{15}=0.26\right)$ by convolving a numerically redshifted $\mathrm{O}$ star spectrum with the UIT filter response and comparing this with its $z=0$ counterpart. No corrections for Galactic extinction have been applied because $E_{B-V} \lesssim 0.01$.

We also photometered a polygonal region $\left(\sim 8 \times 4^{\prime \prime}\right)$ centered on the dust lane visible within the HST image to compare the colors inside and outside this region. The change in color compared with an identical region centered on the optical nucleus is $\Delta(15-V)=$ 0.8 .

\subsection{Other Objects}

There are 10 previously cataloged objects, excluding the $\mathrm{cD}$ galaxy, visible in the UIT image. Five of these are cataloged only in the HST Guide Star Catalog (GSC) while the other five are SAO82997 $\left(m_{15}=14.00 \pm\right.$ $0.07,(15-V)=4.89, \mathrm{~F} 8 \mathrm{~V}), \mathrm{SAO} 83004\left(m_{15}=\right.$ $14.30 \pm 0.10,(15-V)=4.97, \mathrm{~F} 0 \mathrm{~V})$, Abell cluster galaxies \#40 and \#151, Huchra et al. 1990) and $1345.6+2639\left(m_{15}=15.85 \pm 0.10\right.$, (15$V)=0.15$; a Seyfert galaxy). The two stars and the Seyfert galaxy are all point sources. There is no previously published photometry for the two cluster galaxies, but we have es- 
timated their colors based upon their digitized Palomar Sky Survey (DPOSS) images. We find: Abell \#40, $m_{15}=16.4 \pm 0.2$, (15$V) € 3.0$, Abell \#151, $m_{15}=16.6 \pm 0.2$, (15$V)=2.3$. These magnitudes have not been corrected for $K$-dimming or for extinction (external or intrinsic). Both objects are quite bly e for normal cluster galaxies and may be experiencing some star formation. Assuming their UV light is due to young stars we estimate that Abell \#40 and Abell \#151 contain $\sim 8500 \mathrm{O}$ stars. This would imply star formation rates of approximately $1 \mathrm{M}_{\odot} \mathrm{yr}^{-1}$ We note however that their $(15-V)$ colors are similar to those seen in galaxies with "UV upturns" arising from hot EHB stars in the old population (Dorman, O'Connell, \& Rood 19\$5). Therefore, without other evidence for recent star formation ( $\mathrm{H} \alpha$ emission for example) we cannot distinguish between young stars and EHB stars as the primary cause for blue colors.

In addition to these sources there are four uncataloged sources (all are $5 \sigma$ detections) with mean magnitude $\left\langle m_{15}\right\rangle=16.7$. Comparison of their positions with the DPOSS suggest all of these are stars or groups of stars too faint in the visible to be included in the HST GSC. Comparison with deep optical images has proven to be fruitful in identifying faint UIT sources (O'Neil, et al. (1996) which are easily overlooked on the POSS. We expect the same may be true for this image as there are several faint $(2-3 \sigma)$ sources without optical counterparts in the DPOSS.

\section{Discussion and Summary}

To estimate the star formation history or current star formation rate using the UV measurements from UIT we must first determine the nature of the UV light. If the light arises primarily from emission-line gas then it will tell us little about massive star formation. Early IUE spectroscopy by Nørgaard-Nielsen, Jørgensen, \& Hansen (1984)] suggested that the UV light was due to high temperature gas, but later IUE observations did measure a continuum (Hu (1992), Crawford \& Fabian 1993). Moreover, recent optical spectroscopy has found strong evidence of a hot continuum (Allen 1995) at optical wavelengths. Spectroscopy at all wavelengths reveals line ratios from the emission-line gas that are inconsistent with ionization via a nuclear, nonthermal power-law source. The LINER-like spectrum of Abell 1795 is better fit by shock models (van Breugel, Heckman, \& Miley (1984), Hu (1992), Anton 1993) or photoionization by a hot population (Allen 1995). Thus, it seems likely that the bulk of the UV emission in the UIT bandpass does come from a continuum of hot stars which may in turn be responsible for ionizing some of the emission-line gas.

\subsection{Star Formation}

Since at least some portion of the observed $\mathrm{H} \alpha$ and Ly $\alpha$ flux arises from gas photoionized by a UV continuum there must be a significant population of young hot stars, implying either a recent episode of star formation $(t \sim$ few Myr) or continuous star formation. The UIT magnitude listed in Table 3.3 implies a luminosity at $1500 \AA, L_{1500}=1.9 \times 10^{42}$ erg $\sec ^{-1}$. We can estimate the number of O5 stars required to produce the measured UIT flux and then compare that with the expected star formation rate needed to fuel the $\mathrm{H} \alpha$ emission. Using Kurucz model atmospheres (Kurucz 1993), assuming and effective temperature and luminosity of $38,000 \mathrm{~K}$, $\log \left(\frac{L_{b o l}}{L_{\odot}}\right)=5.7$ for a mid O star, and cosmology with $H_{o}=75, q_{o}=0.1$ we re- 
quire $1.8 \times 10^{4}$ stars to match the UIT, $K-$ corrected flux. This represents the minimum number of ionizing stars because no extinction correction has been applied and mid $\mathrm{O}$ stars produce more ionizing flux than early B stars. Without ultraviolet spectroscopic information we cannot further restrict the relative contributions of various stellar types with temperatures greater than $15000 \mathrm{~K}$ to the UIT flux. Adopting the extinction $\left(E_{B-V}=\right.$ 0.14) from $\mathrm{Hu}(1992)$ and assuming a Galactic extinction law (Savage and Mathis 1979) and foreground screen dust model causes the estimated number of $\mathrm{O}$ stars to rise to $5.3 \times 10^{4}$. Recent optical spectroscopy by Allen 1995 predicts $2.4 \times 10^{4} \mathrm{O}$ stars (adjusting to our cosmology) using similar extinction, suggesting that the screen model may be a reasonable approximation for the dust extinction in the galaxy center. The implied star formation rate $(\mathrm{SFR})$ is in the range $8-23 M_{\odot} \mathrm{yr}^{-1}$, depending on the IMF model, with the lower bounds corresponding to a Kennicutt (1983) IMF and the upper bound corresponding to a Miller \& Scalo (1979) IMF. Since some of the $\mathrm{H} \alpha$ emission comes from shocked gas a SFR derived assuming all the emission come from photoionized gas is an upper limit. Therefore, it is likely that the actual SFR is closer to the lower limit in the case of Abell 1795. This SFR is consistent with the predicted star formation rates one calculates from the $\mathrm{H} \alpha$ luminosity $\left(5 M_{\odot} \mathrm{yr}^{-1}\right.$, van Breugel, Heckman, \& Miley (1984), Kennicutt (1983)). These star formation rates are substantially lower than those estimated from recent $\mathrm{X}$-ray analyses $\left(\sim 300 M_{\odot \mathrm{yr}^{-1}}\right.$; Edge, Stewart, \& Fabian 1922, Fabian 1994).

To further test the various scenarios for possible star formation histories we use models based upon (a) a single, instantaneous burst of star formation (b) a single, exponentially decaying burst of star formation (c) continuous, constant star formation (d) a single, instantaneous old burst with smaller, younger bursts superimposed. The first three models are described in more detail in Cornett et al. (1994). The fourth model is simply a scaled superposition of the models in (a). We have plotted color-color diagrams using the four colors $(15-V),(15-U),(U-V),(V-R)$ for each set of the above models to compare with the global colors of the cD galaxy (figure (7). The colors have been corrected for $K$-dimming, but not extinction. Extinction vectors based upon various models for the distribution of the dust are added to the plot. Several models for the star formation history can be ruled out. Models with exponentially decaying star formation and those in which the central population was created in a single burst of age greater than few hundred Myr all have broad-band colors too red to match the $\mathrm{cD}$. Models with some levels of continuous star formation and/or those with recent bursts however can reproduce the colors. The most plausible burst model is one in which an old galaxy $\left(z_{\text {form }}=5\right)$ experiences a Salpeter IMF burst of age $4 \mathrm{Myr}$ and total mass $25 \%$ of the old population, within the photometered aperture. We estimated the mass interior to $r=\sim 25^{\prime \prime}$ by comparing the fraction of light within this radius for an $r^{1 / 4-\text { law galaxy with }}$ its total and assumed a constant $M / L$ ratio of 20 . Since this likely underestimates the central $M / L$ ratio the mass required of our burst to match the $\mathrm{cD}$ colors is an upper limit. The most plausible continuous, constant star formation model which matched the far UV visible colors is one inn which the galaxy has been forming stars at a rate $\sim 5-10 M_{\odot} \mathrm{yr}^{-1}$ over the past 5 Gyr. 


\subsection{Dust Extinction}

The dust lane visible in figure 3 complicates the interpretation of broad-band observations, particularly those involving ultraviolet light. By comparing the measured $I U E$ Ly $\alpha$ flux to $\mathrm{H} \alpha$ flux and assuming suitable values for the intrinsic line flux ratio, $\mathrm{Hu}$ (1992) estimated the intrinsic $E_{B}-V=0.14$, but with a wide possible range $\left(0.2<E_{B-V}<\right.$ 0.22 . Recent optical spectroscopy by Allen 1955 suggests $E_{B-V}=0.22$. The straight line vectors in figure 7 show the effects of dust assuming both the Galactic Savage \& Mathis $1 9 \longdiv { 9 } ( A _ { 1 5 2 0 } = 8 . 3 2 E _ { B - V } )$ and SMC extinction laws. The curved lines (for a dusty galaxy, similar to a spiral and an elliptical galaxy model) are the extinction curves based upon the dust/stellar distribution models of Witt, Thronson \& Capuano 1992. Their models of mixed dust, gas and stars generally produce smaller extinction in the UV than a simple foreground screen models. The location of the galaxy in the color-color diagrams is consistent with systems having continuous star formation over the last 5-10Gyr when combined with the $E_{B-V}=0.14$, Galactic-law screen or dusty galaxy extinction models, or with a system characterized by an old population with a 2-5Myr burst and the same extinction laws. Extinction laws with $E_{B-V}=0.22$ combined with measured $\mathrm{cD}$ colors generally produce values too blue to be consistent with any of our galaxy models.

\subsection{Conclusions}

Our analyses reveal several things. The light at $1520 \AA$ is best characterized by an exponential or disk-like light profile with a scale length of $4 \mathrm{kpc}$. Similarly, the F702W light is best matched by a predominantly disk-like profile but with scale length of $2 \mathrm{kpc}$. The
UV-optical colors match those of models with continuous star formation for $t \sim 5-10 \mathrm{Gyr}$ or those of an old galaxy with a massive, young $(t \sim 4 \mathrm{Myr})$ burst. Both of these properties are consistent with the UV light coming from an accreted population formed in a cluster cooling flow or from an episode of star formation induced by an interaction with a much smaller galaxy. The cooling flow scenario has the following points as supporting evidence (a) line ratios indicative of shock heated gas and (b) the inner surface brightness profile does not show significant disturbances in the UV or optical bands. However, detailed image analyses of deep $U$ imaging by McNamara, et al. (1996b) appears to have revealed possible debris associated with an interaction of the $\mathrm{cD}$ with two small galaxies. These features are too faint to have been detected with the UIT. High resolution images in the UV would help further constrain the nature of the UV bright population.

We wish to thank Brian McNamara for providing us with a $U$-band image of Abell 1795. EPS also thanks Jason Pinkney for helpful comments about photometry of HST images. This research has made use of the NASA/IPAC Extragalactic Database (NED) which is operated by the Jet Propulsion Laboratory, Caltech, under contract with the National Aeronautics and Space Administration. 
TABle 1

Surface Brightness Profile Models

\begin{tabular}{lrr}
\hline \hline \multicolumn{1}{c}{ Model } & Scale Length $\left[{ }^{\prime \prime}\right]$ & \multicolumn{1}{c}{$\chi^{2}$} \\
\hline UV: Disk & $4.5 \pm 0.3$ & 5.4 \\
UV: $r^{\frac{1}{4}}$-law & $41.1 \pm 16.6$ & 18.3 \\
$R:$ Disk a & $3.7 \pm 0.04$ & 4.9 \\
$R: r^{\frac{1}{4}}$-law ${ }^{\text {a }}$ & $142 \pm 32$ & 42.7 \\
$R:$ (composite) Disk $+r^{\frac{1}{4}}$-law + point source & & 0.54 \\
$R$ (composite) Disk component & $2.2 \pm 0.3$ & \\
$R$ (composite) $r^{\frac{1}{4}}$-law component & $0.3 \pm 0.1$ & \\
\hline
\end{tabular}


peak in HST profile. 
TABle 2

Photometric Properties ${ }^{\mathrm{a}}$

\begin{tabular}{lr}
\hline \hline Parameter & \multicolumn{1}{c}{ Value } \\
\hline$m_{c, 15}$ & $15.36 \pm 0.07$ \\
$(15-U)$ & -0.18 \\
$(15-V)$ & 0.53 \\
$(V-R)$ & 0.61 \\
\hline
\end{tabular}

${ }^{a}$ All properties measured within $r=12^{\prime \prime}$ aperture.

${ }^{\mathrm{b}}$ Values include $K$-corrections (see 2.2). 


\section{REFERENCES}

Allen, S. W. 1995, MNRAS, 276, 947

Anton, K. 1993, A\&A, 270, 60

Coleman, Wu, C. C., \& Weedman, D. 1980, ApJ, 43393

Cornett, R. H., O'Connell, R. W., Greason, M. R., Offenberg, J. D., Angione, R. J., Bohlin, R. C., Cheng, K.-P., Roberts, M. S., Smith, A. M., Smith, E. P., Talbert, F. D.\& Stecher, T. P 1994, ApJ, 426, 553

Crawford, C. S., \& Fabian, A. C. 1993, MNRAS, 265, 431

Dorman, B., O'Connell, R. W., \& Rood, R. T. 1995, ApJ, 442, 105

Edge, A.C., Stewart, G.C., \& Fabian, A.C. 1992, MNRAS, 258, 177

Fabian, A.C. 1994, ARA\&A, 32, 277

Ferguson, H. C., et al. 1991, ApJ, 381, L69

Ge, J. P., \& Owen, F. N. 1993, AJ, 105, 778

Heckman, T. M. 1981, ApJ, 250, L59

Hill, J. M., \& Oegerle W. R. 1993, AJ, 106, 831

Holtzman, J. et al. 1995, preprint

Hu, E. M., Cowie, L. L., \& Wang, Z. 1985, ApJS, 47, 447

Hu, E. M. 1992, ApJ, 391, 608

Huchra, J. et al. 1990, ApJS52, 89

Kennicutt, R. C. 1983, ApJ, 272, 54

Kurucz, R. 1993, CD-Rom, No. 13
McKee, J. D., Mushotzky, R.F., Boldt, E. A., Holt, S. S., Marshall, F. E., Pravdo, S. H., \& Serlemitsos, P. J. 1980, ApJ, 242, 843

Merkelijn, J. K. 1972, Australian J. Phys., 25, 451

McNamara, B. R., \& O'Connell, R. W., ApJ, 393, 579

McNamara, B. R., \& O'Connell, R. W., AJ, 105,417

McNamara, B. R., Jannuzi, B. T., Elston, R., Sarazin, C. L., \& Wise, M. 1996, ApJ, in press

McNamara, B. R., Wise, M,, Sarazin, C. L., Jannuzi, B.T., \& Elston, R. 1996, ApJ, preprint

Miller, G. E., \& Scalo, J. M. 1979, ApJS, 41, 513

Nørgaard-Nielsen, H. E., Jørgensen, H. E., \& Hansen, L. 1984, A\&A, 135, L3

O’Neil, K., Bothun, G. D., Smith, E. P., \& Stecher, T. P. 1996, AJ, in press

O'Connell, R. W., et al. 1992, ApJ395, L45

Pinkney J., et al., 1996, BAAS, 27, 1443

Savage, B. D. and Mathis, J. S. 1979 ARA\&A, 17,73

Sargent, W. L. W. 1973, ApJ, 182, L13

Stecher, T. P., et al. 1992 ApJ, 395, L1

van Breugel, W., Heckman, T., \& Miley, G. 1984, ApJ, 276, 79

Witt, A., Thronson, \& Capuano, J, 1992, ApJ, 393, 611

This 2-column preprint was prepared with the AAS 
$\mathrm{IAT}_{\mathrm{EX}}$ macros v4.0. 
Fig. 1.- Full UIT image of the Abell 1795 cluster field centered on the cD galaxy. Other cataloged objects are labeled and discussed in section 2.3. The location and orientation of the HST WFPC aperture used for its observation is overlaid for comparison.

Fig. 2.- $\mathrm{H} \alpha$ contours from van Breugel, Heckman \& Miley (1984) superimposed on a greyscale UIT image of the $\mathrm{cD}$ galaxy. There is no far-UV counterpart to the extended $\mathrm{H} \alpha$ emission seen at $r=22^{\prime \prime}$

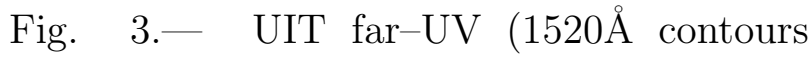
starting at $\sim 3 \sigma_{\text {sky }}$ increasing linearly by $1.1 \times 10^{-18} \mathrm{erg} \mathrm{s}^{-1} \mathrm{~cm}^{-2} \AA^{-1}$ per contour superimposed on the HST F555W image. The bulk of the cD galaxy lies outside the PC chip boundaries. Hence the image is stretched to emphasize the innermost galaxy core. The contour colors are wrapped for clarity of contrast with the greyscale image.

Fig. 4.- UIT far-UV $(1520 \AA$ contours starting at $\sim 2 \sigma_{\text {sky }}\left(7.2 \times 10^{-18} \mathrm{erg} \mathrm{s}^{-1} \mathrm{~cm}^{-2} \AA^{-1}\right.$ $\operatorname{arcsec}^{-2}$ and incremented linearly overlaid on the VLA $3.6 \mathrm{~cm}$ map from Ge \& Owen (1993). The radio image has been smoothed to the UIT resolution.

Fig. 5.- Upper Panel: Surface brightness profile (magnitudes $\operatorname{arcsec}^{-2}$ ) in the $1520 \AA$ band along with best fitting disk and elliptical galaxy models. Lower panel: Color profiles for the galaxy over the region defined by the surface brightness profile.

Fig. 6. - Surface Brightness profile (mag $\operatorname{arcsec}^{-2}$ for the HST F702W image of the central part of Abell 1795.

Fig. 7.- Color-color diagrams showing the location of the $\mathrm{cD}$ along with several models of star formation histories. Symbols have the following meaning: (•) Single generation, instantaneous bursts of star formation with the age of the burst (in Myr) under the symbol, $(\triangle)$ Single generation, exponentially decaying star formation with the decay time (Myr) next to the symbol, ( $\square$ ) Constant, continuous star formation, beginning at the time listed under the symbol, (*) Single, 4 Myr old burst added to an old population (see 3.1), ("cD") Abell 1795 measured colors. Tracks indicating the effects of extinction are superimposed (see 3.2). The errors in the $\mathrm{cD}$ colors are smaller that the letters used to label the point itself. 

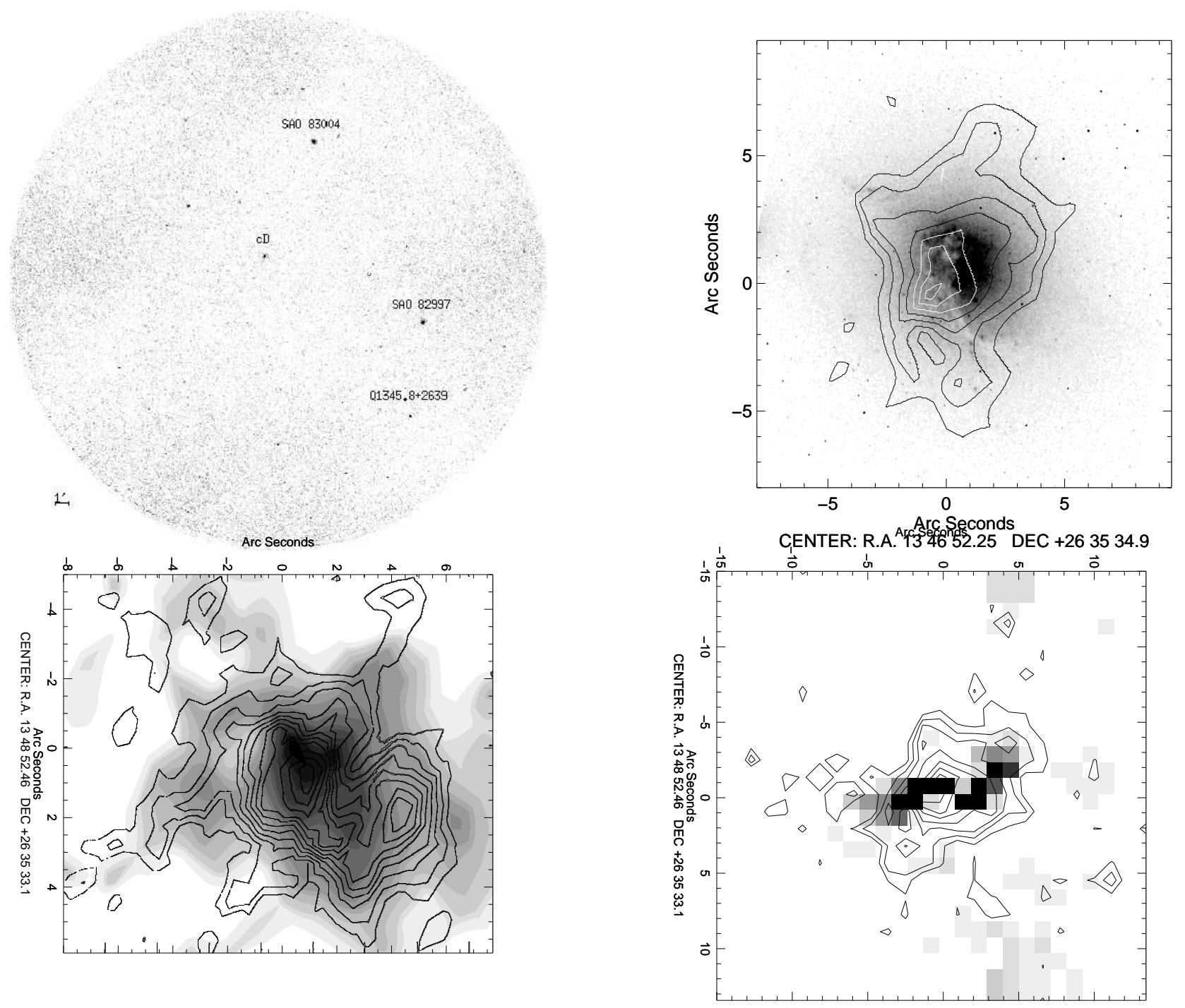

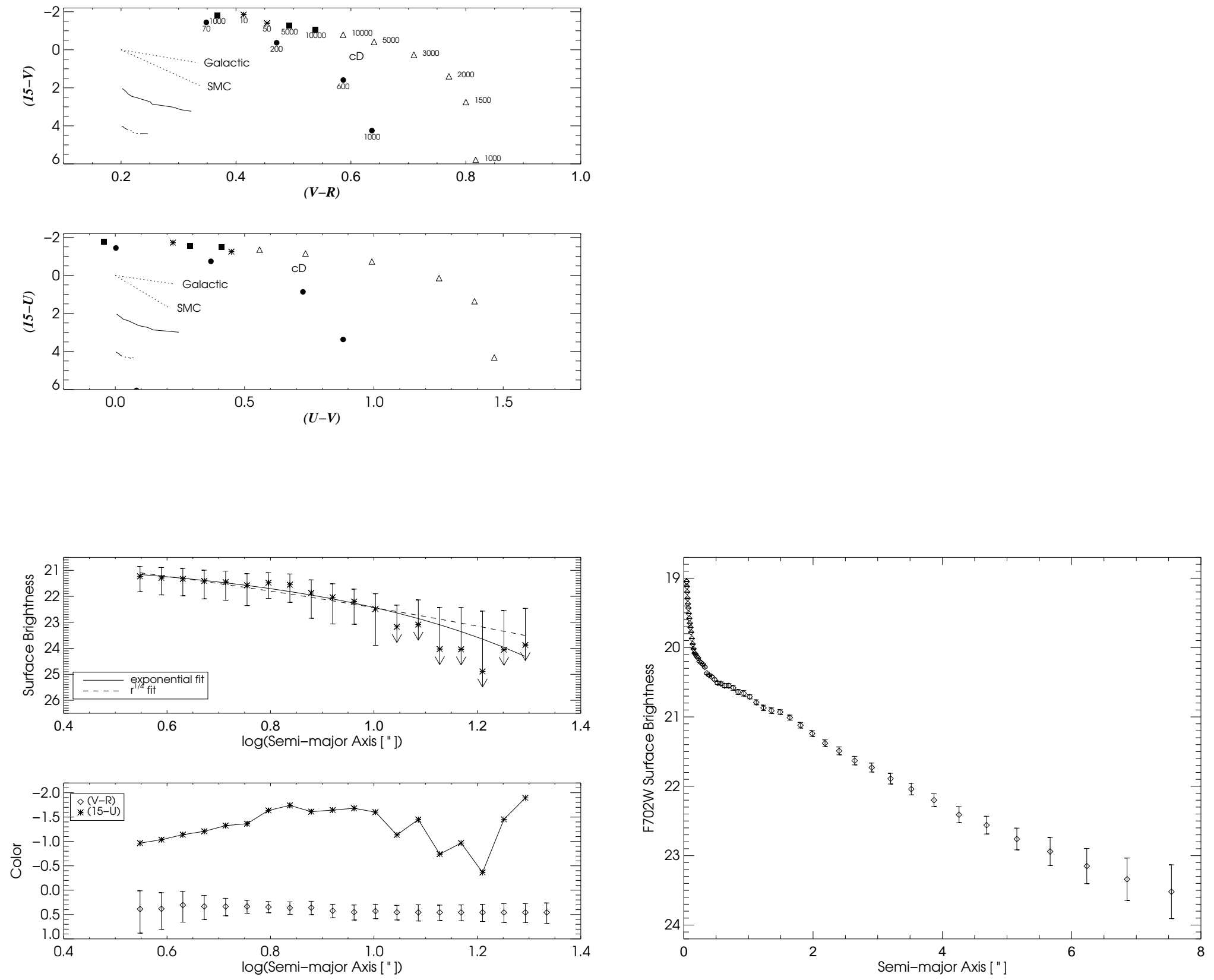